\title{
Web-based Teaching Radio Interferometer for Africa
}

\author{
Claude Carignan ${ }^{1,2}$ and Yannick Libert ${ }^{1}$ \\ ${ }^{1}$ Department of Astronomy, University of Cape Town, P.B. X3, Rondebosch 7701, South Africa \\ ${ }^{2}$ Observatoire d'Astrophysique de l'Université de Ouagadougou, Burkina Faso \\ email: ccarignan@ast.uct.ac.za
}

\begin{abstract}
This presentation describes the web-based Teaching Radio Interferometer being built on the campus of the University of Cape Town, in South Africa, to train the future users of the African VLBI (Very Long Baseline Interferometry) Network (AVN).
\end{abstract}

Keywords. instrumentation: interferometers, techniques: interferometric

\section{Overview}

Practical training for the future use of the African VLBI Network (AVN) or any VLBI experiment starts by understanding the basic principles of radio observations and radio interferometry. The aim of this project is to build a basic interferometer that could be used remotely via a web interface from any country on the African continent. This should turn out as a much less expensive and much more efficient way to train AVN researchers from SKA partner countries to the principles of radio astronomy and to interferometric data analysis. The idea is based on the EUHOU (European Hands-On Universe) project, already very successful in Europe. The former EUHOU manager, Dr Yannick Libert, arrived for a 3 years postdoc with Prof Claude Carignan at the University of Cape Town to implement the same project on the African continent (AHI: African Hands-on Interferometry). Besides the use of AHI for the AVN researchers, this web-based system could be used be any undergraduate program on radio astronomical techniques across the African continent as the EUHOU is used all across Europe.

Our goal is to build a robust instrument that can be used remotely in classrooms on the African continent for years to come. The dishes will be two $2.3 \mathrm{~m}$ parabolas hooked up to equatorial mounts. The mounts of the telescopes are computerised and the signal will be sent over a coaxial cable to the receiver box. This box will be linked to a fast fiber optic switch so that it can be accessible by a server installed in the Astronomy department that will provide access to the instrument through the Web. The receivers will be designed for $21 \mathrm{~cm}$ observations and coupled with a fast processor that will allow interferometric acquisition between the 2 antennas. A server will be installed for storing the data and sharing the control of the instrument over the Web. This project is funded by the AVN training programme. It aims at training students to radio observations techniques all over Africa. The teaching interferometer received R420 000 to be developed.

With the advent of SKA (Square Kilometre Array) and its precursors KAT-7 and MeerKAT, and the development of the AVN, the field of radio astronomy is undergoing a massive development. To sustain this growth, we need to develop efficient training programmes. Successful realisation, demonstration and fruitful usage of the facility on the University of Cape Town premises during 2015 can lead to realisation of similar facilities in the Universities of SKA partner countries. Botswana and Mauritius already showed interest in replicating this project. 